\title{
Fibras solúveis e insolúveis de verduras, tubérculos e canela para uso em nutrição clínica
}

\author{
Soluble and insoluble fibres of vegetables, tubers and cinnamon for use in enteral nutrition
}

\author{
Edma Maria de ARAÚJO'*, Hilary Castle de MENEZES ${ }^{1}$, Julien Mironescu TOMAZINI ${ }^{1}$
}

\begin{abstract}
Resumo
Dietas enterais com alimentos convencionais são usadas para obter individualização e menor custo. No entanto, para melhorar o teor de fibras alimentares, o valor nutritivo e a confiabilidade, mais estudos são necessários. A proposta desse estudo foi estudar fontes de fibra alimentar de abóbora, agrião, canela em pó, couve-flor, inhame e tomate, para uso em nutrição enteral ou suplemento nutricional oral. Esses alimentos foram escolhidos por conter fibras alimentares e outros elementos considerados funcionais (isotiocianatos, indóis, licopeno, carotenoides, clorofila). Foram formuladas preparações com os alimentos em solução de água e adicionadas a uma formulação enteral domiciliar. Foram determinadas fibras solúvel e insolúvel, a composição centesimal dos alimentos e a atividade de água das formulações. Os alimentos foram acrescentados à formulação enteral domiciliar em quantidades de $100 \mathrm{~g} / 2000 \mathrm{kcal} / 2 \mathrm{~L}$ e à água $150 \mathrm{~g} / 2000 \mathrm{kcal} / 2 \mathrm{~L}$. Essas quantidades equivalem a duas porções diárias de cada alimento. A maior quantidade de fibra alimentar (base úmida) foi de canela (50,11\%), sendo o teor dos outros alimentos: abóbora 1,76\%; agrião $0,85 \%$; couve-flor 1,51\%; inhame 1,56\%; e tomate 1,00\%. Os valores de atividades de água ficaram entre 0,988 e 0,999, indicando que as dietas são suscetíveis à contaminação microbiana, sendo mais seguro consumir essas dietas imediatamente após o preparo.
\end{abstract}

Palavras-chave: fibra alimentar; nutrição enteral; suplemento nutricional oral; atividade de água.

\begin{abstract}
Enteral diets containing conventional foods are used to reduce costs and obtain individualization. However, more studies are required to improve their dietary fibre content, the nutritive value, and reliability. This study proposed to study the dietary fibres of different vegetables and spices such as pumpkin, watercress, powdered cinnamon, cauliflower, yam, and tomato for use in enteral nutrition or in oral nutritional supplements. These foods were chosen since they contain dietary fibres and other elements considered to be functional (isothiocyanate, indoles, lycopene, carotene, and chlorophyll). They were prepared in water solution and added to a homemade enteral formula. The soluble and insoluble fibres, proximate composition, and water activity (Aw) were determined. The foods were added to the homemade enteral formula in the proportion of $100 \mathrm{~g} / 2000 \mathrm{kcal} / 2 \mathrm{~L}$ and in the proportion of $150 \mathrm{~g} / 2000 \mathrm{kcal} / 2 \mathrm{~L}$ to the water. The largest amount of dietary fibre (wet basis) was found in the cinnamon (50.11\%). The other foods results were: pumpkin, $1.76 \%$, watercress $0.85 \%$, cauliflower $1.51 \%$, yam $1.56 \%$, and tomato $1.00 \%$. The Aw values were between 0.988 and 0.999 , and so it indicates that the preparations can therefore be used in enteral diets, but they should be consumed soon after preparation to assure microbiological safety.

Keywords: dietary fibre; enteral nutrition; oral nutritional supplement; water activity.
\end{abstract}

\section{Introdução}

$\mathrm{O}$ uso de dietas enterais (DE) tem evoluído intensamente nos últimos anos. A nutrição enteral (NE) é o tratamento de escolha para pacientes que não podem manter sua ingestão alimentar oral adequada e que tenham o trato gastrointestinal funcionante (BAXTER; CECCONELLO; PINOTTI, 1997; HAFEZ, 2006; HEBUTERNE, et al., 2003; WAITZBERG, 1997). A NE tem sido recomendada por ser uma forma mais fisiológica de alimentação (SILVA, et al., 2005).

As fibras alimentares (FA) estão sendo consideradas importantes no tratamento de pacientes com DE. Dentre as aplicações das FA na nutrição clínica, pode-se citar o gerenciamento da função do intestino, a intensificação de sua integridade e função absortiva, a manutenção da barreira intestinal e a normalização da microflora intestinal (BAXTER, et al., 2000; CATALANI, et al., 2003). As FA são componentes de alimentos convencionais e, quando possível, devem fazer parte da alimentação diária.

As propriedades funcionais das fibras são determinadas pela inter-relação entre estruturas e características físico-químicas (MONGEAU; BRASSARD; VERDIER, 1989). O conhecimento dessas propriedades funcionais das fibras pode ser explorado por profissionais da saúde e servir como base para recomendações dietéticas mais específicas. Para tanto, torna-se necessário identificar as frações solúveis e insolúveis (TORRES, et al., 2006).

A internação domiciliar é uma nova modalidade de acompanhamento do paciente, cada vez mais expressiva em nosso meio (DIAS, 2001). Tratar o doente sob regime domiciliar tem se

Recebido para publicação em 24/10/2004

Aceito para publicação em 8/9/2008 (002956)

${ }^{1}$ Núcleo de Estudos e Pesquisas em Alimentação - NEPA, Universidade Estadual de Campinas - UNICAMP, Rua Albert Einstein, 291, CEP 13083-852, Campinas - SP, Brasil,

E-mails: edmarau@unicamp.br; hilary@mpc.com.br; jumt@fea.unicamp.br

${ }^{*}$ A quem a correspondência deve ser enviada 
mostrado mais vantajoso, tanto sob o ponto de vista do doente e da família, como também das entidades pagadoras. Se, por um lado, ocorre a humanização do atendimento e manutenção do doente em convívio familiar, por outro, destacam-se as vantagens econômicas advindas da otimização de leitos hospitalares, menor custo do tratamento e melhor qualidade de vida do paciente. $\mathrm{O}$ intuito é atender o doente em regime ambulatorial domiciliar com indicação de terapia nutricional, visando otimizar recursos da instituição sem prejuízos aos cuidados nutricionais do doente (BAXTER, 2002; GOLDWASSER; GOLDWASSER, 2001; RILEY; MANTHORPE, 2003; SINGER, 2001; STEPHAN; BRITO; MONTONE, 2003). No entanto, esse tipo de tratamento requer uma abordagem multidisciplinar com critérios definidos por uma equipe de atendimento domiciliar (SINGER, 2001).

As fórmulas para NE, preparadas com alimentos convencionais, reduzem os custos do tratamento dos pacientes, permitindo a extensão desse tipo de terapia nutricional às classes socioeconômicas mais baixas da sociedade. Contudo, o monitoramento do valor nutritivo deste tipo de dieta é de suma importância para melhorar a qualidade e a segurança do valor nutritivo.

Assim, nesse estudo, a proposta foi estudar fontes de fibra alimentar de abóbora, agrião, canela em pó, couve-flor, inhame e tomate, para uso em nutrição enteral ou suplemento nutricional oral. Esses alimentos foram escolhidos por conter fibras alimentares e outros elementos considerados funcionais (isotiocianatos, indóis, licopeno, carotenoides, clorofila) (SOUSA; SOUSA NETO; MAIA, 2003; VAN DUYN; PIVONKA, 2000), para serem adicionados a uma formulação enteral domiciliar (SUNDFELD, 2000), para uso em NE ou suplemento nutricional oral, e obter dietas reformuladas com melhor qualidade e maior teor de FA.

\section{Material e métodos}

\subsection{Matéria-prima}

Alimentos experimentais: agrião (Rorippa Nasturtium-aquaticum (L.) (Hayek); abóbora (Cucurbita spp); canela em pó (Cinnamomum Aromaticum); couve-flor (Brassica Oleracea L. var Botrytis L.); inhame (Colocasia Esculenta (L.) (Schott); e tomate (Lycopersicum Esculentum Mill).
Alimentos constituentes da formulação enteral domiciliar (SUNDFELD, 2000): Soymilke sabor natural (extrato de soja em pó desengordurado, açúcar, maltodextrina, vitaminas A, B1, B2, B6, B12, C, D, E, K, niacina, ácido pantotênico, ácido fólico e biotina, minerais como cálcio, ferro, fósforo, magnésio, zinco, cobre, iodo, sódio e manganês, aroma natural de baunilha e goma guar como espessante), ovo vermelho tipo médio, óleo de soja e Nidex $^{\oplus}$ (maltodextrina). Os alimentos (Tabela 1) foram adquiridos em supermercados de Campinas, SP, no estágio de maturação em que são rotineiramente comercializados e consumidos.

\subsection{Métodos}

\section{Reformulação das dietas para nutrição enteral}

Os alimentos experimentais foram adicionados a uma formulação enteral usada em nutrição domiciliar (SUNDFELD, 2000).

Na padronização das formulações, foi considerado um volume de $2 \mathrm{~L}$ e valor calórico aproximado de $2000 \mathrm{kcal}$, quantidade normalmente usada para consumo de um dia. As quantidades de alimentos foram adequadas para se obter um percentual $20 \%$ de sólidos, (CARVALHO; COUTO; SALES, 1992), considerado adequado para gotejamento gravitacional.

$\mathrm{O}$ valor nutricional das formulações com os alimentos experimentais foi calculado com dados da literatura (IBGE, 1977; MENDEZ, et al., 1995) e informações nutricionais das embalagens (Tabela 2).

Tabela 1. Quantidades de alimentos experimentais e de alimentos da formulação enteral domiciliar usados para compor as formulações (g/2 L/2000 kcal).

\begin{tabular}{lc}
\hline $\begin{array}{c}\text { Alimentos } \\
\text { experimentais }(\mathrm{g})\end{array}$ & $\begin{array}{c}\text { Alimentos da fórmula } \\
\text { enteral domiciliar }(\mathrm{g})\end{array}$ \\
\hline Abóbora 100 & Soymilke $^{\circledR} 200$ \\
Agrião 100 & Ovo inteiro 50 \\
Canela em pó 25 & Clara 25 \\
Couve-flor 100 & Óleo de soja 18 \\
Inhame 100 & Nidex $170 / 185$ \\
Tomate 100 & \\
\hline
\end{tabular}

Formulação enteral domiciliar (SUNDFELD, 2000).

Tabela 2. Valor nutricional das formulações com os alimentos adicionados à formulação enteral domiciliar, calculados com dados da literatura - $2 \mathrm{~L}$.

\begin{tabular}{|c|c|c|c|c|c|c|}
\hline \multicolumn{7}{|c|}{ Formulações com os alimentos experimentais } \\
\hline Nutrientes & Abóbora & Agrião & Canela & Couve-flor & Inhame & Tomate \\
\hline Calorias (kcal) & 1983,62 & 1959,62 & 2002,87 & 1970,62 & 2001,62 & 1958,62 \\
\hline Umidade (g) & 155,46 & 159,36 & 69,66 & 156,56 & 139,79 & 160,96 \\
\hline Lipídios (g) & 70,10 & 70,20 & 70,55 & 70,20 & 69,90 & 70,76 \\
\hline Carboidratos $(\mathrm{g})$ & 267,48 & 260,98 & 277,68 & 264,18 & 271,98 & 262,34 \\
\hline
\end{tabular}

Formulação enteral domiciliar (SUNDFELD, 2000). Literatura (IBGE, 1977; MENDEZ, et al., 1995). Informações nutricionais das embalagens (Nestlé Brasil Ltda para Nidex ${ }^{\circledR}$; Olvebra Industrial S.A. para Soymilke ${ }^{\circledR}$. 
Foram mantidas as quantidades de Soymilke ${ }^{\circledR}$, ovo inteiro, clara e óleo de soja e acertadas as quantidades de Nidex ${ }^{\bullet}$ e de alimentos (abóbora, agrião, canela em pó, couve-flor, inhame e tomate) (Tabela 1) para se obter dietas com os percentuais de proteínas, lipídios e carboidratos adequados em relação ao valor calórico total (BAXTER, et al., 2000) e com maior quantidade de FA possível. Para a dieta com inhame, com maior teor de amido e valor calórico, foi utilizada a proporção de $170 \mathrm{~g}$ de Nidex ${ }^{\circledR}$ para $2 \mathrm{~L}$ de dieta; para os outros alimentos, com menor valor calórico, foram utilizados $180 \mathrm{~g}$ para $2 \mathrm{~L}$.

Após estabelecidas as quantidades de alimentos, as dietas foram preparadas e foram realizados os testes de gotejamento, para verificação das características do escoamento.

\section{Formulações para suplemento nutricional oral}

Para as dietas orais, para ser ingeridas por boca com o auxílio de um "canudinho", foram feitos testes com as preparações para estabelecer as melhores quantidades de cada alimento em relação ao volume total e obter o máximo possível de FA. Os testes foram feitos preparando os alimentos adicionados à formulação enteral domiciliar (SUNDFELD, 2000). Após o preparo, as dietas foram colocadas em copos descartáveis e testadas com "canudinhos" (canudos de plástico Pingo-lin milk-250, com diâmetro de $5 \mathrm{~mm}$ ).

\section{Preparo das formulações enterais e orais}

As dietas foram preparadas (ARAÚJO; GALEAZZI, 1999; ARAÚJO; MENEZES, 2005 e 2006) em laboratório (ambiente similar a um domícilio), em panela de pressão (12 psi, cap. $4 \mathrm{~L}$, Colck $^{\oplus}$, Brasil). Os alimentos foram cortados em pedaços pequenos, colocados em água fria e levados à cocção em fogo baixo durante 20 minutos. Em seguida, a panela foi retirada do fogo e resfriada em água fria até temperatura ambiente. A preparação foi então triturada e homogeneizada em liquidificador (Migiclean, Arno ${ }^{\oplus}$, São Paulo, Brasil) junto com o Soymilke ${ }^{\circledR}$ o Nidex $^{\circledR}$ e o óleo, peneirada (peneira de nylon, tela plana, com aberturas de $1 \mathrm{~mm}$ de diâmetro), colocada em recipiente de vidro e mantida em refrigerador $\left(5^{\circ} \mathrm{C}\right)$ até o dia seguinte, para testes de escoamento por capilar.

\section{Determinações físico-químicas e físicas das dietas}

A homogeneidade e estabilidade das preparações foram observadas por inspeção visual após preparo, acondicionamento em béquer de vidro e refrigeração $\left(5^{\circ} \mathrm{C}\right)$ por 24 horas (WAITZBERG, 1985).

Os testes de gotejamento (gotas/minuto) foram realizados pelo método gravitacional, usando $200 \mathrm{~mL}$ de dieta em frascos plásticos $\left(\right.$ Darrow $\left.^{\circ}\right)$ descartáveis, flexíveis, com capacidade de $500 \mathrm{~mL}$ e equipo para administração de DE (Laboratório B. Braun ${ }^{\circ}$.

$\mathrm{O} \mathrm{pH}$ foi determinado em amostras de $12 \mathrm{~mL}$ (à temperatura de $23^{\circ} \mathrm{C}$ e sem adição de óleo) com o uso de pHmetro (Tecnal ${ }^{\circ}-2$, Piracicaba, Brasil).

\section{Composição centesimal}

A determinação da composição centesimal de cada amostra foi feita em duplicata para obtenção do valor médio. As amostras foram liofilizadas após cocção e congelamento. Lipídios totais foram determinados pelo método de Bligh e Dyer (1959), extração com solventes, seguido de determinação gravimétrica. Proteína bruta pelo método de semi Kjeldahl (AOAC, 1984). A conversão da porcentagem do nitrogênio total em proteína bruta foi feita com o fator de 6,25 (SGARBIERI, 1996). Fibras solúveis e insolúveis foram determinadas pelo tratamento com enzimas digestivas (amilase, pepsina/pancreatina), precipitação alcoólica da fração solúvel, incineração e determinação gravimétrica segundo o método de (ASP et al., 1983). As cinzas foram quantificadas por carbonização e incineração das amostras em mufla $\left(550^{\circ} \mathrm{C}\right)$ e a umidade em estufa $\left(105^{\circ} \mathrm{C}\right)$ (IAL, 1985). Carboidratos totais (CHOT) (g/100 g de alimento) foram calculados pela seguinte fórmula: CHOT = 100 - (proteínas + lipídios + cinzas + umidade + FA total) (FAO, 1995). No cálculo dos macronutrientes, foram usados os fatores de conversão de $4 \mathrm{kcal}$ para carboidrato e proteína e de $9 \mathrm{kcal}$ para lipídios (BRASIL, 2003).

\section{Análise estatística}

Os resultados das análises de fibra foram apresentados em valores médios e desvios padrão para fibras insolúveis e solúveis dos alimentos, obtidos através de uma análise de variância considerando um nível de significância de 5\%. O pacote estatístico utilizado para a realização do teste foi o SAS (Statistical Analysis System).

\section{Determinação da atividade de água}

As determinações das atividades de água $(\mathrm{Aw})$ foram realizadas usando o equipamento AquaLab CX-2 (Decagon Devices Inc., USA), acoplado a um banho termostático para manter constante a temperatura durante as medições. Essas condições permitem fazer medidas de Aw em valores de temperatura compreendidos entre 20 e $43^{\circ} \mathrm{C}$. Para as presentes determinações, a temperatura foi mantida em $(25,0 \pm 0,4){ }^{\circ} \mathrm{C}$. A calibragem do AquaLab CX-2 para a determinação da Aw foi feita utilizando-se água destilada (Milli-Q) para $\mathrm{Aw}=1,000$ e soluções saturadas de cloreto de sódio, cloreto de potássio, cloreto de lítio e nitrato de magnésio, de acordo com a metodologia do manual do equipamento. $\mathrm{O}$ AquaLab é um determinador rápido da Aw, realizando cada leitura em, aproximadamente, 5 minutos. Em geral, as medidas realizadas em duplicata apresentam uma diferença de, no máximo, \pm 0,001 unidades de Aw. Por ser uma determinação física não destrutiva, usando volumes pequenos, é recomendado efetivar a determinação em triplicata para maior precisão.

As amostras das dietas ( 3 a $5 \mathrm{~mL}$ ) obtidas logo após o preparo foram colocadas em um porta-amostras incluído no circuito de água circulante por aproximadamente 10 minutos antes das medições, para equilibrar a temperatura em $25^{\circ} \mathrm{C}$.

\section{Resultados e discussão}

\subsection{Formulações para nutrição enteral}

A quantidade de cada alimento adicionada às formulações foi de $100 \mathrm{~g} / 2 \mathrm{~L}$ de dieta com aproximadamente $2000 \mathrm{kcal}$, com 
exceção da canela com $25 \mathrm{~g} / 2 \mathrm{~L}$. Todas as formulações ficaram com aproximadamente, $20 \%$ de sólidos totais (CARVALHO; COUTO; SALES, 1992).

Com essas quantidades de ingredientes, foram obtidas soluções com um escoamento adequado para gotejamento gravitacional, de 60 a 80 gotas por minuto (WAITZBERG, 1995) para todos os alimentos. Apesar das dietas com alimentos convencionais apresentarem viscosidade mais elevada (MITNE, 2000) do que as dietas industrializadas, esses volumes ficaram adequados com os padrões da literatura (WAITZBERG, 1995) para administração por gotejamento gravitacional.

Para as preparações com os alimentos experimentais cozidos em solução de água, as melhores proporções obtidas foram de $100 \mathrm{~g} / 400 \mathrm{~mL}$ para abóbora e inhame e de $100 \mathrm{~g} / 300 \mathrm{~mL}$ para a couve-flor. As soluções com a canela, o agrião e o tomate, preparadas com água, não foram aprovadas para utilização enteral, por que não apresentou homogeneidade adequada.

Devem ser realizados mais estudos para avaliar a eficácia, a tolerância e a aceitação da canela em pó para utilização em nutrição clínica.

$\mathrm{O} \mathrm{pH}$ das dietas com os alimentos adicionados à formulação enteral domiciliar (SUNDFELD, 2000) - em temperatura de $25^{\circ} \mathrm{C}$-, apresentaram resultados acima de seis para todos os alimentos: abóbora 6,84; agrião 6,65; canela 6,55; couve-flor 6,75 ; inhame 6,28 ; tomate 6,85 .

\subsection{Resultados das análises da composição centesimal}

$\mathrm{Na}$ Tabela 3, estão apresentados os dados das análises de composição centesimal dos alimentos liofilizados.

A Tabela 3 mostra os resultados das determinações da composição centesimal (base seca) e os teores médios de FA insolúvel e solúvel dos alimentos. Os resultados apresentados em valores médios e desvios padrão para fibras insolúveis e solúveis dos alimentos foram obtidos através de uma análise de variância considerando um nível de significância de 5\%.

Os testes estatísticos mostraram que as quantidades médias de fibras insolúveis, de uma maneira geral, do tomate, do agrião e da couve-flor, da abóbora e do agrião diferem estatisticamente ao nível de 5\% de significância. A canela e o inhame diferem estatisticamente dos outros alimentos, para esse mesmo parâmetro, comprovando que esses alimentos apresentam as maiores e as menores quantidades de fibra insolúvel.

Quanto às fibras solúveis, os resultados estatísticos mostraram que há diferença significativa ao nível de $5 \%$ entre as médias de couve-flor e tomate, inhame e tomate, abóbora e inhame; canela e inhame, agrião e canela. Apesar de a couve-flor apresentar a maior quantidade média de fibra solúvel, estatisticamente, esse valor médio é igual ao valor médio de fibra solúvel para o tomate e a abóbora.

A Tabela 4 mostra os resultados das análises de composição centesimal dos alimentos experimentais (base úmida).

Tabela 3. Composição centesimal (g/100 g, base seca ), média e desvio padrão dos resultados das análises químicas dos alimentos liofilizados.

\begin{tabular}{|c|c|c|c|c|c|c|}
\hline \multicolumn{7}{|c|}{ Alimentos liofilizados } \\
\hline Nutrientes & Abóbora (g) & Agrião (g) & Canela (g) & Couve-flor (g) & Inhame $(\mathrm{g})$ & Tomate $(\mathrm{g})$ \\
\hline Calorias $(\mathrm{kcal})^{1}$ & 363,58 & 341,55 & 173,97 & 312,04 & 355,66 & 293,42 \\
\hline Umidade $(\mathrm{g})^{2}$ & $83,40 \pm 0,65$ & $92,22 \pm 0,81$ & $10,38 \pm 0,11$ & $92,47 \pm 0,61$ & $80,82 \pm 0,87$ & $95,25 \pm 0,03$ \\
\hline Proteínas $(\mathrm{g})^{3}$ & $11,71 \pm 0,07$ & $38,22 \pm 0,16$ & $3,59 \pm 0,02$ & $19,00 \pm 0,08$ & $5,87 \pm 0,16$ & $12,31 \pm 0,05$ \\
\hline Lipídios $(\mathrm{g})^{3}$ & $6,30 \pm 0,16$ & $7,83 \pm 0,02$ & $2,21 \pm 0,00$ & $5,20 \pm 0,16$ & $1,06 \pm 0,04$ & $1,34 \pm 0,15$ \\
\hline Carboidratos $(\mathrm{g})^{4}$ & 65,10 & 118,96 & 34,93 & 47,52 & 324,04 & 232,12 \\
\hline Fibra insolúvel ${ }^{3,5}(\mathrm{~g})$ & $7,27^{\mathrm{d}} \pm 0,28$ & $9,59^{c, d} \pm 0,33$ & $54,18^{\mathrm{a}} \pm 0,77$ & $15,77^{\mathrm{b}, \mathrm{c}} \pm 0,92$ & $5,18^{\mathrm{e}} \pm 0,52$ & $17,79^{b} \pm 0,25$ \\
\hline Fibra solúvel $^{3,5}(\mathrm{~g})$ & $3,33^{\mathrm{a}, \mathrm{b}} \pm 0,13$ & $1,40^{\mathrm{d}} \pm 0,43$ & $1,73^{c, d} \pm, 16$ & $4,30^{\mathrm{a}} \pm 0,48$ & $2,95^{b, c} \pm 0,12$ & $3,21^{\mathrm{a}, \mathrm{b}} \pm 0,29$ \\
\hline Cinzas $(\mathrm{g})^{3}$ & $6,38 \pm 0,11$ & $13,41 \pm 0,27$ & $3,36 \pm 0,01$ & $8,21 \pm 0,18$ & $4,28 \pm 0,14$ & $7,32 \pm 0,27$ \\
\hline
\end{tabular}

${ }^{1} \mathrm{kcal}$ = proteína e carboidrato multiplicados por 4 e lipídios por 9 (BRASIL, 2003). ${ }^{2}$ Umidade: com alimentos crus, canela em pó desidratada. ${ }^{3}$ Proteínas, lipídios, fibras e cinzas com alimentos cozidos e liofilizados. ${ }^{4}$ Por diferença 100 g-(umidade + proteínas + lipídios + fibra alimentar total + cinzas) (FAO, 1995). ${ }^{5}$ Médias com letras diferentes indicam que os valores são estatisticamente diferentes ao nível de $5 \%$ de significância. Média das duplicatas.

Tabela 4. Composição centesimal (g/100 g, base úmida), média dos dados das análises químicas dos alimentos liofilizados.

\begin{tabular}{|c|c|c|c|c|c|c|}
\hline \multicolumn{7}{|c|}{ Alimentos liofilizados } \\
\hline Nutrientes & Abóbora (g) & Agrião (g) & Canela (g) & Couve-flor (g) & Inhame (g) & Tomate (g) \\
\hline Calorias $(\mathrm{kcal})^{1}$ & 60,36 & 25,56 & 155,90 & 23,59 & 68,20 & 13,98 \\
\hline Umidade $(\mathrm{g})^{2}$ & 83,40 & 92,22 & 10,38 & 92,47 & 80,82 & 95,25 \\
\hline Proteínas $(\mathrm{g})^{3}$ & 1,94 & 2,97 & 3,21 & 1,43 & 1,12 & 0,58 \\
\hline Lipídios $(\mathrm{g})^{3}$ & 1,04 & 0,60 & 1,98 & 0,39 & 0,20 & 0,06 \\
\hline Carboidratos $(\mathrm{g})^{4}$ & 10,81 & 2,32 & 31,31 & 3,59 & 15,48 & 2,78 \\
\hline Fibra insol. $(\mathrm{g})^{3, *}$ & 1,21 & 0,74 & 48,56 & 0,32 & 0,99 & 0,85 \\
\hline Fibra sól. $(\mathrm{g})^{2, *}$ & 0,55 & 0,11 & 1,55 & 1,18 & 0,57 & 0,15 \\
\hline Cinzas $(\mathrm{g})^{2}$ & 1,05 & 1,04 & 3,01 & 0,61 & 0,82 & 0,34 \\
\hline
\end{tabular}

${ }^{1} \mathrm{Kcal}=$ proteína e carboidrato multiplicados por 4 e lipídios por 9 (BRASIL, 2003). ${ }^{2}$ Umidade: com alimentos crus; canela em pó desidratada. ${ }^{3}$ Proteínas, lipídios, fibras e cinzas com alimentos cozidos e liofilizados. ${ }^{4}$ Por diferença 100 g-(umidade + proteína + lipídios + fibra alimentar total + cinzas) (FAO, 1995). *Fibra insolúvel e solúvel. Média das duplicatas. 
A maior quantidade de FA total é representada pela canela com $50,11 \%$. Para os outros alimentos, as quantidades de FA foram de 1,76\% em abóbora, 0,85\% em agrião, 1,51\% em couveflor, $1,56 \%$ em inhame, $1,00 \%$ em tomate. As quantidades de FA dos alimentos adicionadas em $2 \mathrm{~L}$ de dieta ficaram bem abaixo das recomendações diárias de $25 \mathrm{~g}$ /dia para uma dieta de $2000 \mathrm{kcal}$ (CATALANI, et al., 2003). No entanto, as quantidades adicionadas representam duas porções diárias de cada alimento em $2000 \mathrm{kcal}$ - valor calórico, geralmente, utilizado para um dia. A suplementação de FA na DE também é recomendada em pequenas quantidades em caráter preventivo para manter o equilíbrio das funções intestinais (WAITZBERG, 2004). Segundo BAXTER, et al., (2000) as recomendações de fibras para os pacientes em terapia nutricional enteral não são numericamente definidas. A sugestão da literatura é iniciar com uma quantidade mínima e evoluir segundo a tolerância digestiva do enfermo. Não há necessidade de serem atingidas as recomendações diárias para indivíduos adultos normais $(25$ a $30 \mathrm{~g} /$ dia), considerando-se a imobilidade e a mudança de hábito alimentar significativo que acomete a maioria dos doentes em NE (BAXTER, et al., 2000).

O conteúdo de fibras alimentares das formulações industrializadas varia entre 4 a $20 \mathrm{~g}$ de fibras por litros de dieta (BAXTER, et al., 2000). Nas dietas reformuladas com os alimentos foram acrescentados alguns valores menores, no entanto, sabe-se que as dietas formuladas com alimentos in natura apresentam maior viscosidade (MITNE, 2000), o que dificulta a inclusão de maiores quantidades de alimentos com fibras.

A presença de FA na DE é importante para manter a microflora colônica e o equilíbrio de $\mathrm{pH}$, proteger a mucosa intestinal e estimular o sistema imunológico local (BAXTER, 2001).

Considerando-se a importância das fibras na alimentação clínica, é importante obter e disponibilizar dados atualizados para viabilizar a utilização mais segura e obter melhores resultados nas recomendações dietéticas mais específicas.

\subsection{Formulações para suplemento nutricional oral}

As quantidades de cada alimento adicionadas à formulação enteral domiciliar (SUNDFELD, 2000), para uso em suplemento nutricional oral, foram de $150 \mathrm{~g} / 2 \mathrm{~L}$ para abóbora, agrião, couve-flor e inhame e $200 \mathrm{~g} / 2 \mathrm{~L}$ para o tomate. Para a canela, duas quantidades podem ser usadas, $30 \mathrm{~g} / 2 \mathrm{~L}$ e $40 \mathrm{~g} / 2 \mathrm{~L}$, isto é, soluções menos e mais concentradas, ambas com sabor agradável, principalmente se servidas geladas. No entanto, pequenas quantidades são recomendadas para iniciar a utilização e depois aumentar de acordo com a tolerância individual.

Todos os alimentos foram cozidos durante 20 minutos em panela de pressão, tempo suficiente para cozinhar e possibilitar a homogeneização, resultando em partículas de tamanho reduzido, permitido assim um aproveitamento total, ou seja, sem resíduos retidos na peneira.
Tabela 5. Atividades de água da abóbora, agrião, canela em pó, couveflor, inhame e tomate cozidos com água e com a formulação enteral domiciliar. Medições realizadas em temperatura de $(25,0 \pm 0,4)^{\circ} \mathrm{C}$.

\begin{tabular}{lcccc}
\hline \multicolumn{1}{c}{ Dietas } & Aw1 & Aw2 & Aw3 & $\bar{A} w \pm \Delta \mathrm{Aw}$ \\
\hline Abóbora + água & 0,998 & 0,999 & 0,998 & $0,998 \pm 0,001$ \\
Abóbora + dieta & 0,993 & 0,993 & 0,994 & $0,993 \pm 0,001$ \\
Agrião + água & 0,996 & 0,999 & 0,996 & $0,997 \pm 0,002$ \\
Agrião + dieta & 0,988 & 0,988 & 0,992 & $0,989 \pm 0,002$ \\
Canela + água & 0,992 & 0,992 & 0,993 & $0,992+0,001$ \\
Canela + dieta & 0,992 & 0,992 & 0,992 & $0,992 \pm 0,000$ \\
Couve-flor + água & 0,994 & 0,994 & 0,994 & $0,994 \pm 0,000$ \\
Couve-flor + dieta & 0,989 & 0,989 & 0,989 & $0,989 \pm 0,000$ \\
Inhame + água & 0,997 & 1,000 & 1,000 & $0,999 \pm 0,002$ \\
Inhame + dieta & 0,996 & 0,996 & 0,995 & $0,996 \pm 0,001$ \\
Tomate + água & 0,994 & 0,995 & 0,993 & $0,994 \pm 0,001$ \\
Tomate + dieta & 0,988 & 0,989 & 0,988 & $0,988 \pm 0,001$ \\
\hline
\end{tabular}

Formulação enteral domiciliar (SUNDFELD, 2000).

\section{Resultados da determinação da atividade de água}

Os resultados das análises da determinação da Aw estão apresentados na Tabela 5.

Pode-se observar, na Tabela 5, que a Aw tanto dos vegetais cozidos com água quanto com a dieta, são altas, entre 0,988 e 0,99 . Os resultados estão de acordo com o esperado, já que na literatura (BOBBIO; BOBBIO, 2001), os valores de Aw para vegetais são em torno de 0,99 . Os resultados mostram que os alimentos podem ser utilizados, porém cuidados adequados com as condições de higiene no preparo, armazenamento e utilização das dietas devem ser seguidos rigorosamente. As dietas devem ser consumidas logo após o preparo, com exceção das formulações com canela, agrião e tomate, preparadas com água para dieta enteral, que não apresentou homogeneidade adequada.

\section{Conclusões}

Foi possível adicionar fibras dos alimentos experimentais na formulação enteral domiciliar. As quantidades de cada alimento são de $100 \mathrm{~g} / 2 \mathrm{~L} / 2000 \mathrm{kcal}$ para abóbora, agrião, couve-flor, inhame, tomate e $25 \mathrm{~g} / 2 \mathrm{~L} / 2000 \mathrm{kcal}$ para canela em pó. As quantidades equivalem a duas porções diárias de cada alimento e representam teores de fibra alimentar (base úmida) de 1,76\% para abóbora, $0,85 \%$ para agrião, $50,11 \%$ para canela, $1,51 \%$ para couve-flor, $1,56 \%$ para inhame e $1,00 \%$ para o tomate. No entanto, os valores de atividade de água entre 0,988 e 0,999, indicam que as dietas são suscetíveis à contaminação microbiana, sendo mais seguro consumir essas dietas imediatamente após o preparo. $\mathrm{O}$ uso de técnicas adequadas de higienização dos alimentos e utensílios é imprescindível no preparo e utilização das dietas.

Os alimentos que podem ser utilizados cozidos com água são a abóbora, a couve-flor e o inhame.

\section{Agradecimentos}

Os autores agradecem à FAPESP - Fundação de Amparo à Pesquisa do Estado de São Paulo, o auxílio financeiro ao projeto de pesquisa e à Empresa Novozymes a doação da enzima termamyl. 


\section{Referências bibliográficas}

ARAÚJO, E. M.; GALEAZZI, M. A. M. Inventores: Fórmulas para nutrição enteral com alimentos convencionais. BR. nº. PI 9905419-1. UNICAMP, Campinas, 12 de Novembro de 1999.

ARAÚJO, E. M.; MENEZES, H. C. Formulações com alimentos convencionais para nutrição enteral ou oral. Ciência e Tecnologia de Alimentos, v. 26, n. 3, p. 533-538, 2006.

. Composição centesimal, lisina disponível e digestibilidade in vitro de proteínas de fórmulas para nutrição oral ou enteral. Ciência e Tecnologia de Alimentos, v. 25, n. 4, p. 768-771, 2005.

ASP, N. G. et al. Rapid enzymatic assy of insoluble and soluble dietary fiber. Journal of Agricultural and Food Chemistry, v. 31, n. 3, p. 476-482, 1983.

ASSOCIATION OF OFFICIAL ANALYTICAL CHEMISTS - AOAC. Official methods of analysis. 14 ed. Washington D.C., 1984. p. 1141.

BAXTER, Y. C. Fibras alimentares: nutriente funcional na prevenção e tratamento da obstipação intestinal. Boletim da Sociedade Brasileira de Nutrição Parenteral e Enteral, n. 32, p. 18-19, 2001.

BAXTER, Y. C. Estudo econômico de programa de terapia nutricional enteral domiciliar em pré-operatório de cirurgia digestiva em instituição pública. São Paulo, 2002. Dissertação (Doutorado em Nutrição e Cirurgia) - Faculdade de Medicina, Universidade de São Paulo - USP. (Apud: Newsletter Nutritotal). Disponível em: <http:// nutritotal.com.br>. Acesso em: 13 de Janeiro de 2006.

BAXTER, Y. C.; CECCONELLO, I.; PINOTTI, H. W. Nutrição enteral domiciliar: introdução e bases técnicas. Revista Brasileira de Nutrição Clínica, v. 12, n. 1, p. 8-16, 1997.

BAXTER, Y. C. et al. Critérios de decisão na seleção de dietas enterais. In: WAITZBERG, D. L. (Ed.). Nutrição oral, enteral e parenteral na prática clínica. 3 ed. São Paulo: Atheneu, 2000. Cap. 41, p. 659-676.

BLIGH, E. G.; DYER, W. J. A rapid method of total lipid extraction and purification. Canadian Journal of Biochemistry and Physiology, v. 37, n. 8, p. 911-917, 1959.

BOBBIO, P. A.; BOBBIO, F. O. Química do processamento de alimentos. 3 ed. São Paulo: Varela, 2001. p. 5-8.

BRASIL. Ministério da Saúde. Agência Nacional de Vigilância Sanitária. Resolução - RDC no 360, de 23 de dezembro de 2003. Brasília, DF. Disponível em: <http://www.anvisa.gov.br/legis/resol/2003/ rdc/360_03rdc.htm\#>. Acesso em: 13 de Janeiro de 2008.

CARVAlHO, E. B.; COUTO, C. M. F.; SALES, T. R. A. Manual de Suporte Nutricional. Rio de Janeiro: Médica e Científica Ltda., 1992. Cap. 4, p. 41-63.

CATALANI, L. A. et al. Fibras alimentares. Revista Brasileira de Nutrição Clínica, v. 18, n. 4, p. 178-182, 2003.

DIAS, A. C. R. Abordagem farmacêutica na nutrição enteral. Revista Brasilira de Home Care, n. 74, p. 9, 2001.

FOOD AND AGRICULTURE ORGANIZATION - FAO. Informe del taller CTPD sobre produccion y manejo de datos de composicion química de alimentos en nutricion de america latina. Santiago do Chile, 1995. p. 36.

GOLDWASSER, D.; GOLDWASSER, R. Adaptação do suporte nutricional enteral do paciente com doença crônica em assistência domiciliar. Revista Brasileira de Home Care, n. 79, p. 10, 2001.

HAFEZ, V. C. B. Nutrição enteral domiciliar $\times$ qualidade de vida. Newsletter Nutritotal Nutrição Enteral XVI. Disponível em: <http:// nutritotal.com.br>. Acesso em: 13 de Janeiro de 2006.
HEBUTERNE, X. et al. Home enteral nutrition in adults: a European Multicentre Survey. Clinical Nutrition, v. 22, n. 3, p. 261-266, 2003.

INSTITUTO BRASILEIRO DE GEOGRÁFIA E ESTATÍSTICA - IBGE. Estudo nacional de despesa familiar. Tabela de composição de alimentos. 4 ed. Rio de Janeiro, 1977.

INSTITUTO ADOLFO LUTZ. Normas analíticas. 3 ed. São Paulo, 1985.

MENDEZ, M. H. M. et al. Tabela de composição de alimentos. Nitéroi: Universidade Federal Fluminense, 1995.

MITNE, C. Preparações não-industrializadas para nutrição enteral. In: WAITZBERG, D. L (Ed.). Nutrição oral, enteral e parenteral na prática clínica. 3 ed. São Paulo: Atheneu, 2000. Cap. 38, p. 629-640.

MONGEAU, R.; BRASSARD, R.; VERDIER, P. Measurement of dietary fiber in a total diet study. Journal of Food Composition and Analysis, v. 2, n. 4, p. 317-326, 1989.

RILEY, A. J.; MANTHORPE, J. The impact of home enteral tube feeding in everyday life: a qualitative study. Health Social - Care Community, v. 11, n. 5, p. 415-422, 2003.

SGARBIERI, V. C. Proteínas em alimentos protéicos. São Paulo: Varela, 1996. Cap. IV, p. 337-381.

SILVA, C. A. B. et al. Reações in vitro entre drogas de liberação programada e dietas enterais. Revista Brasileira de Nutrição Clínica, v. 20, n. 4, p. 255-258, 2005.

SINGER, S. D. et al. Suplementação oral: podemos evitar a utilização de sonda no idoso? Revista Brasileira de Home Care, n. 72, p. 22, 2001.

SOUSA, P. H. M.; SOUSA NETO, M. A.; MAIA, G. A. Componentes funcionais nos alimentos. Boletim da Sociedade Brasileira de Ciência e Tecnologia de Alimentos, v. 37, n. 2, p. 127-135, 2003.

STEPHAN, A. B.; BRITO, S.; MONTONE, M. Perfil nutricional dos pacientes com terapia nutricional enteral em assistência domiciliária. Revista Brasileira de Home Care, n. 93, p. 26-28, 2003.

SUNDFELD, L. C. R. Manual de Dietas. Campinas: Divisão de Nutrição e Dietética, Hospital de Clínicas da UNICAMP, 2000.

TORRES, G. F. et al. Efeito do processo hidrotérmico sobre o teor de fibra alimentar em hortaliças. Boletim do Centro de Pesquisa e Processamento de Alimentos, v. 24, n. 2, p. 237-346, 2006.

VAN DUYN, M. A. S.; PIVONKA, E. Overviw of the health benefits of fruit and vegetable consumption for the dietetics professinal: Selected literature. Journal of the American Dietetic Association, v. 100, n. 12, p. 1511-1521, 2000.

WAITZBERG, D. L. Nutrição Enteral e Parenteral na Prática Clínica. In: CHAAYA, A. P. et al. Suporte nutricional enteral em ambiente domiciliar: Via e método de administração e complicações após 1 ano de prestação de serviços. Revista Brasileira de Nutrição Clínica, v. 12, n. 1, p. 21-26, 1997.

Eficácia e tolerância de uma nova formulação dietética enteral em doentes desnutridos. Revista da Associação Médica Brasileira, v. 31, n. 11-12, p. 214-221, 1985.

. Nutrição enteral e parenteral na prática clínica. 2 ed. São Paulo: Atheneu, 1995.

. Fibras em nutrição enteral. Newsletter Nutritotal Nutrição Enteral XLII. Disponível em: <http://nutritotal.com.br>. Acesso em: 22 de Janeiro de 2004. 